\title{
Stabilization of Networked Control Systems with Clock Offsets
}

\author{
Masashi Wakaiki, Kunihisa Okano, and João P. Hespanha
}

\begin{abstract}
We consider the stabilization of networked control systems with time-invariant clock offsets between the sensors and the controllers. Clock offsets are modeled as parametric uncertainty and we provide necessary and sufficient conditions for the existence of a single controller that is capable of stabilizing the closed loop for every clock offset in a given range. For scalar systems, we obtain the maximum length of the offset interval for which the system can be stabilized by a single linear time-invariant controller. We compare the offset bounds that would be allowed by specific classes of controllers for scalar systems.
\end{abstract}

\section{INTRODUCTION}

In networked control systems, real-time data on plants is not available at the controller side due to transmission and/or computation delays. Since these delays are timevarying and unpredictable, generally, the sensors transmit data with time-stamps to provide information on the time at which the data was collected as in [1]. The problem is that the clocks in the sensors and at the controller may differ. Hence protocols to establish synchronization have been actively studied as surveyed in [2], [3], and synchronization using Global Positioning Systems (GPS) or radio clocks have been utilized in practical systems. However, synchronizing clocks over networks has fundamental limits [4]. Recent works [5], [6] have also shown that synchronization technologies based on GPS signals may be vulnerable against malicious attacks.

In this paper, we consider a networked control system in which the internal clock of the sensor is not synchronized with that of the controller. Our objective is to determine how large the error between the clocks can be without compromising the existence of a single linear time-invariant controller that stabilizes the closed loop. Our previous work [7] has studied a similar stabilization problem with any casual controllers, based on the state estimation method under data-rate constraints.

We formulate the feedback stabilization with clock offsets as the problem of stabilizing systems with parameter uncertainty and provide a necessary and sufficient condition for the existence of a single controller that stabilizes the closed loop for every value of the clock offset in a given range. For general-order systems, from this condition we can only obtain computationally efficient conditions that are either necessary or sufficient. For the special case of scalar systems,

This material is based upon work supported by the National Science Foundation under Grant No. CNS-1329650. M. Wakaiki acknowledges The Kyoto University Foundation for the support of this work. K. Okano is supported by JSPS Postdoctoral Fellowships for Research Abroad.

The authors are with the Center for Control, Dynamical-systems and Computation (CCDC), University of California, Santa Barbara, CA 93106-9560 USA (e-mail: masashiwakaiki@ece.ucsb.edu; kokanodece.ucsb.edu; hespanha@ece.ucsb.edu). the condition leads to the exact bound on the clock offset that can be allowed for stability. To compare this bound obtained by using the class of linear time-invariant stabilizers, we also obtain the exact bound that would be allowed by a static stabilizers, and derive a sufficient condition on offset ranges for the existence of 2-periodic static stabilizers stabilizing the closed-loop system.

The remainder of the paper is organized as follows. Section II gives the system we stabilize and presents the problem formulation. In Section III, we consider generalorder systems and derive a necessary condition and a sufficient condition for the stabilization problem with clock offsets. For scalar systems, we derive the exact bound on the permissible clock offset in Section IV. Section V is devoted to the comparison of the bound under general linear time-invariant stabilizers with that under specific classes of stabilizers for scalar systems.

Notation and definitions: Let $\mathbb{Z}_{+}$be the set of nonnegative integers. Let $\mathbb{D}, \overline{\mathbb{D}}$, and $\mathbb{T}$ denote the open unit disc $\{z \in \mathbb{C}:|z|<1\}$, the closed unit disc $\{z \in \mathbb{C}:|z| \leq 1\}$, and the unit circle $\{z \in \mathbb{C}:|z|=1\}$, respectively.

We denote by $\mathcal{H}^{\infty}$ the space of all bounded holomorphic scalar-valued functions in $\mathbb{D}$. $\mathcal{R} \mathcal{H}^{\infty}$ denotes the subspace of $\mathcal{H}^{\infty}$ consisting of all real-rational functions. Let us denote the field of fraction of $\mathcal{H}^{\infty}$ by $\mathcal{F}^{\infty}$.

For a commutative ring $R, \mathbf{M}(R)$ denotes the set of matrices with entries in $R$, of whatever order. For $M \in$ $\mathbf{M}(\mathbb{C})$, let $\|M\|$ be the induced 2-norm, which is equal to the largest singular value of $M$. For $G \in \mathbf{M}\left(\mathcal{H}^{\infty}\right)$, the $\mathcal{H}^{\infty}$. norm is defined as $\|G\|_{\infty}=\sup _{z \in \mathbb{D}}\|G(z)\|$.

We say that $C \in \mathbf{M}\left(\mathcal{F}^{\infty}\right)$ stabilizes $P \in \mathbf{M}\left(\mathcal{F}^{\infty}\right)$ if $(I+P C)^{-1}, C(I+P C)^{-1}$, and $(I+P C)^{-1} P$ belong to $\mathbf{M}\left(\mathcal{H}^{\infty}\right)$, and we call $C$ a stabilizer of $P$.

A pair $(N, D)$ in $\mathbf{M}\left(\mathcal{H}^{\infty}\right)$ is said to be right coprime if the Bezout identity $X N+Y D=I$ holds for some $X, Y \in$ $\mathbf{M}\left(\mathcal{H}^{\infty}\right) . P \in \mathbf{M}\left(\mathcal{F}^{\infty}\right)$ admits a right coprime factorization if there exist $D, N \in \mathbf{M}\left(\mathcal{H}^{\infty}\right)$ such that $P=N D^{-1}$ and the pair $(N, D)$ is right coprime.

Similarly, a pair $(\tilde{D}, \tilde{N})$ in $\mathbf{M}\left(\mathcal{H}^{\infty}\right)$ is right coprime if the Bezout identity $\tilde{N} \tilde{X}+\tilde{D} \tilde{Y}=I$ holds for some $\tilde{X}, \tilde{Y} \in$ $\mathbf{M}\left(\mathcal{H}^{\infty}\right) . P \in \mathbf{M}\left(\mathcal{F}^{\infty}\right)$ admits a left coprime factorization if there exist $\tilde{D}, \tilde{N} \in \mathbf{M}\left(\mathcal{H}^{\infty}\right)$ such that $P=\tilde{N} \tilde{D}^{-1}$ and the pair $(\tilde{D}, \tilde{N})$ is left coprime.

If $P$ is a scalar-valued function, we use the expressions coprime and coprime factorization.

\section{Problem Statement}

Consider the following plant:

$$
\dot{x}(t)=A x(t)+B u(t),
$$




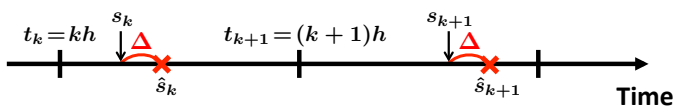

Fig. 1: Sampling instants $s_{k}$, reported time-stamps $\hat{s}_{k}$, and updating instants $t_{k}$ of the zero-order hold

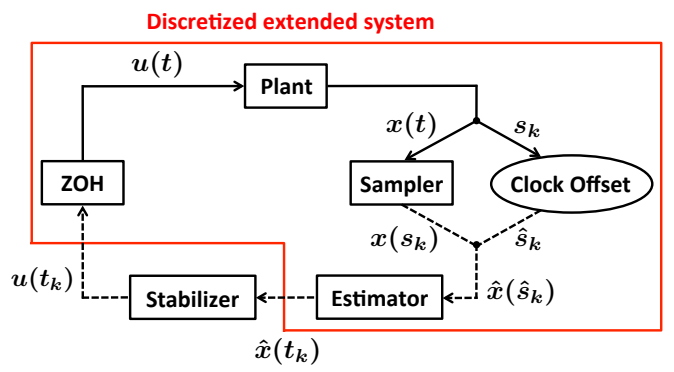

Fig. 2: Closed-loop system with clock offsets

where $x(t) \in \mathbb{R}^{n}$ and $u(t) \in \mathbb{R}^{m}$ are the state and the input of the plant, respectively.

Let $s_{0}, s_{1}, \ldots$ be sampling instants. The sensor observes the state $x\left(s_{k}\right)$ and sends it to the controller together with a time-stamp. However, since the sensor and the controller share no global clock, the time-stamp typically includes an unknown offset with respect to the controller clock, which makes $s_{k}$ uncertain in the controller side. In this paper, we assume that the offset is fixed, that is, $s_{k}$ can be written by the time-stamp $\hat{s}_{k}$ reported by the sensor in the following way: $s_{k}=\hat{s}_{k}-\Delta\left(k \in \mathbb{Z}_{+}\right)$for some unknown constant $\Delta \in \mathbb{R}$. This means that there is a time-invariant offset between the sensor and the controller but no difference in their frequencies, or that this difference is negligible for the time-scales of interest.

The control signal is assumed to be piecewise constant and updated periodically at times $t_{k}=k h\left(k \in \mathbb{Z}_{+}\right)$with values $u_{k}$ computed by a controller: $u(t)=u_{k}$ for $t \in\left[t_{k}, t_{k+1}\right)$. While the control updates are assumed periodic, the true sampling times $s_{k}$ and the reported sampling times $\hat{s}_{k}$ may not be periodic. However, we do assume that both $s_{k}$ and $\hat{s}_{k}$ do not fall behind $t_{k}$ by more than $h$ and that the controller side receives the data $x\left(s_{k}\right)$ and $\hat{s}_{k}$ from the sensor by the next control-updating time $t=t_{k+1}$. This assumption is formally stated as follows.

Assumption 2.1: Fix $h>0$. For $k \in \mathbb{Z}_{+}, t_{k+1}-t_{k}=$ $h$ and $s_{k}, \hat{s}_{k} \in\left[t_{k}, t_{k+1}\right)$. Furthermore, $x\left(s_{k}\right)$ and $\hat{s}_{k}$ are available to the controller by $t=t_{k+1}$.

Fig. 1 shows the timing diagram of the sampling instants, the reported time-stamps, and updating instants of the control inputs.

Using the received state $x\left(s_{k}\right)$ and the reported time-stamp $\hat{s}_{k}$, the controller can estimate the plant state in the following way:

$$
\dot{\hat{x}}(t)=A \hat{x}(t)+B u(t), \quad \hat{x}\left(\hat{s}_{k}\right)=x\left(s_{k}\right) \quad(k \in \mathbb{N}),
$$

where $\hat{x} \in \mathbb{R}^{n}$ is the estimated state. This estimate leads to the discretized extended system in Fig. 2, with state and input given by

$$
\xi_{k}=\left[\begin{array}{c}
x\left(t_{k}\right)-\hat{x}\left(t_{k}\right) \\
\hat{x}\left(t_{k}\right)
\end{array}\right], \quad u_{k}=u\left(t_{k}\right),
$$

respectively, which evolves according to

$$
\xi_{k+1}=F_{\Delta} \xi_{k}+G_{\Delta} u_{k}, \quad y_{k}=H_{\Delta} \xi_{k},
$$

where $p:=e^{A h}, \theta:=e^{-A \Delta}-I$, and

$$
\begin{aligned}
F_{\Delta} & :=\left[\begin{array}{cc}
-p \theta & -p \theta \\
p(I+\theta) & p(I+\theta)
\end{array}\right] \\
G_{\Delta} & :=\left[\begin{array}{c}
p\left(\int_{0}^{h} e^{-A \tau} d \tau-(I+\theta) \int_{0}^{h-\Delta} e^{-A \tau} d \tau\right) B \\
p(I+\theta) \int_{0}^{h-\Delta} e^{-A \tau} d \tau B
\end{array}\right] \\
H_{\Delta} & :=\left[\begin{array}{ll}
0 & I
\end{array}\right] .
\end{aligned}
$$

The objective of the present paper is to obtain lower and upper bounds on the clock offset $\Delta$ under which there exists a single linear time-invariant stabilizer of (II.2) for every value of $\Delta$ in the corresponding range. To obtain these bounds, in what follows we consider the problem below.

Problem 2.2: Given an interval $(\underline{\Delta}, \bar{\Delta})$, determine if there exists a single stabilizer of the extended system (II.2) for every $\Delta \in(\underline{\Delta}, \bar{\Delta})$.

Our second assumption is stabilizability and detectability for the existence of stabilizers.

Assumption 2.3: For all $\Delta \in(\underline{\Delta}, \bar{\Delta}),\left(F_{\Delta}, G_{\Delta}, H_{\Delta}\right)$ is stabilizable and detectable.

Remark 2.4: In Problem 2.2, we consider the stabilization of only the extended discrete-time system (II.2). However, we can easily see that if the stabilization is achieved by a finite-dimensional stabilizer, then we have not only $x\left(t_{k}\right)$, $\hat{x}\left(t_{k}\right) \rightarrow 0(k \rightarrow \infty)$ but also $x(t), \hat{x}(t) \rightarrow 0(t \rightarrow \infty)$.

\section{Analysis via Simultaneous Stabilization}

We first consider a general simultaneous stabilization problem not limited to the system introduced in Section II. Consider the family of plants $P_{\theta} \in \mathbf{M}\left(\mathcal{R} \mathcal{F}^{\infty}\right)$ parametrized by $\theta \in S$, where $S$ is a nonempty set. Assume that we have a doubly coprime factorization of $P_{\theta}$ over $\mathcal{R} \mathcal{H}^{\infty}$ :

$$
\left[\begin{array}{cc}
Y_{\theta} & X_{\theta} \\
-\tilde{N}_{\theta} & \tilde{D}_{\theta}
\end{array}\right]\left[\begin{array}{cc}
D_{\theta} & -\tilde{X}_{\theta} \\
N_{\theta} & \tilde{Y}_{\theta}
\end{array}\right]=I
$$

where $P_{\theta}=D_{\theta}^{-1} N_{\theta}$ and $P_{\theta}=\tilde{N}_{\theta} \tilde{D}_{\theta}^{-1}$ are a right coprime factorization and a left coprime factorization, respectively.

The following theorem gives a necessary and sufficient condition for simultaneous stabilization:

Theorem 3.1 ([8], [9]): Given a nonempty set $S$, let (III.1) hold for every $\theta \in S$. Let $\theta_{0} \in S$. Define $S_{+}:=$ $S \backslash\left\{\theta_{0}\right\}$ and

$$
\left[\begin{array}{c}
U_{\theta} \\
V_{\theta}
\end{array}\right]:=\left[\begin{array}{cc}
Y_{\theta_{0}} & X_{\theta_{0}} \\
-\tilde{N}_{\theta_{0}} & \tilde{D}_{\theta_{0}}
\end{array}\right]\left[\begin{array}{c}
D_{\theta} \\
N_{\theta}
\end{array}\right] \quad\left(\theta \in S_{+}\right) .
$$

Then $\left(V_{\theta}, U_{\theta}\right)$ is right coprime for every $\theta \in S_{+}$. Moreover, there exists a stabilizer of $P_{\theta}$ for every $\theta \in S$ if and only if there exists $Q \in \mathbf{M}\left(\mathcal{R} \mathcal{H}^{\infty}\right)$ such that for all $\theta \in S_{+}$,

$$
\left(U_{\theta}+Q V_{\theta}\right)^{-1} \in \mathbf{M}\left(\mathcal{R} \mathcal{H}^{\infty}\right) .
$$


Such a stabilizer is given by

$$
C:=\left(Y_{\theta_{0}}-Q \tilde{N}_{\theta_{0}}\right)^{-1}\left(X_{\theta_{0}}+Q \tilde{D}_{\theta_{0}}\right) .
$$

It is often not easy to verify the existence of $Q$ with (III.3) in a computationally efficient fashion. To address this challenge, in the remainder of this section we derive computationally attractive conditions that are either necessary or sufficient (but not both). Later in Section IV, we shall explore the specific structure of the system in (II.3) to derive a condition that is both necessary and sufficient, but only applies to scalar systems. However, for the rest of this section, we continue to consider a general process $P_{\theta}$ with factorization in (III.1).

We start by providing a necessary condition that follows from a well-known result on the simultaneous stabilization of two processes via the parity interlacing property.

Corollary 3.2: Given a nonempty set $S$, let (III.1) hold for every $\theta \in S$. Let $\theta_{0}, \theta_{1} \in S$ and define

$$
\left[\begin{array}{c}
U_{\theta_{1}} \\
V_{\theta_{1}}
\end{array}\right]:=\left[\begin{array}{cc}
Y_{\theta_{0}} & X_{\theta_{0}} \\
-\tilde{N}_{\theta_{0}} & \tilde{D}_{\theta_{0}}
\end{array}\right]\left[\begin{array}{c}
D_{\theta_{1}} \\
N_{\theta_{1}}
\end{array}\right] .
$$

If there exists a stabilizer of $P_{\theta}$ for all $\theta \in S$, then for each $\theta_{0}, \theta_{1} \in S, V_{\theta_{1}} U_{\theta_{1}}^{-1}$ satisfies the parity interlacing property, that is, the number of poles of $V_{\theta_{1}} U_{\theta_{1}}^{-1}$ (counted according to their McMillan degrees) between any pair of real blocking zeros of $V_{\theta_{1}} U_{\theta_{1}}^{-1}$ in $\overline{\mathbb{D}}$ is even.

Proof: If $V_{\theta_{1}} U_{\theta_{1}}^{-1}$ does not satisfy the parity interlacing property, then $P_{\theta_{1}}$ and $P_{\theta_{2}}$ do not have a common stabilizer [9, Section 5.4]. Hence there exists no stabilizer of $P_{\theta}$ for all $\theta \in S$.

The next result gives a sufficient condition for (III.3) to hold under certain assumptions on $D_{\theta}$ and $N_{\theta}$. For the system (II.2), Proposition 3.5 facilitates verifying the assumptions of the theorem and gives an explicit formula of $L$ and $f$ in (III.5).

Theorem 3.3: Given a nonempty set $S$, let (III.1) hold for every $\theta \in S$. Let $\theta_{0} \in S$ and define $S_{+}:=S \backslash\left\{\theta_{0}\right\}$. Assume that $D_{\theta}=D_{\theta_{0}}$ for all $\theta \in S$, and that we can write

$$
N_{\theta}(z)-N_{\theta_{0}}(z)=L(z) f(\theta),
$$

where $L \in \mathbf{M}\left(\mathcal{R F}^{\infty}\right)$ and $f(\theta) \in \mathbf{M}(\mathbb{R})$ for $\theta \in S$. Let

$$
\gamma>\inf _{Q \in \mathbf{M}\left(\mathcal{R} \mathcal{H}^{\infty}\right)}\left\|\left(X_{\theta_{0}}+Q \tilde{D}_{\theta_{0}}\right) L\right\|_{\infty} .
$$

If $\|f(\theta)\|<1 / \gamma$ for $\theta \in S$ and if $Q \in \mathbf{M}\left(\mathcal{R} \mathcal{H}^{\infty}\right)$ achieves $\left\|\left(X_{\theta_{0}}+Q \tilde{D}_{\theta_{0}}\right) L\right\|_{\infty}<\gamma$, then $Q$ satisfies (III.3) and hence $C$ in (III.4) stabilizes $P_{\theta}$ for every $\theta \in S$.

Proof: We define $U_{\theta}$ and $V_{\theta}$ as in (III.2). If $D_{\theta}=D_{\theta_{0}}$, then the Bezout identity $Y_{\theta_{0}} D_{\theta_{0}}+X_{\theta_{0}} N_{\theta_{0}}=I$ leads to

$$
U_{\theta}=I+X_{\theta_{0}}\left(N_{\theta}-N_{\theta_{0}}\right) .
$$

In addition, since $\tilde{D}_{\theta_{0}}^{-1} \tilde{N}_{\theta_{0}}=N_{\theta_{0}} D_{\theta}^{-1}$, we obtain

$$
V_{\theta}=-\tilde{D}_{\theta_{0}}\left(\tilde{D}_{\theta_{0}}^{-1} \tilde{N}_{\theta_{0}}-N_{\theta} D_{\theta}^{-1}\right) D_{\theta}=\tilde{D}_{\theta_{0}}\left(N_{\theta}-N_{\theta_{0}}\right) \text {. }
$$

Hence $U_{\theta_{0}}+Q V_{\theta}=I+\left(X_{\theta_{0}}+Q_{0} \tilde{D}_{\theta_{0}}\right)\left(N_{\theta}-N_{\theta_{0}}\right)$. In conjunction with the small gain theorem, the above equation shows that if

$$
\left\|\left(X_{\theta_{0}}+Q \tilde{D}_{\theta_{0}}\right)\left(N_{\theta}-N_{0}\right)\right\|_{\infty}<1 \quad(\theta \in S),
$$

then (III.3) holds for all $\theta \in S$. From the assumption (III.5),

$$
\left\|\left(X_{\theta_{0}}+Q \tilde{D}_{\theta_{0}}\right)\left(N_{\theta}-N_{\theta_{0}}\right)\right\|_{\infty} \leq\left\|\left(X_{\theta_{0}}+Q \tilde{D}_{\theta_{0}}\right) L\right\|_{\infty}\|f(\theta)\| .
$$

Hence if $\|f(\theta)\|<1 / \gamma$ for $\theta \in S$, then $P_{\theta}$ is simultaneously stabilizable by $C$ in (III.4) from Theorem 3.1.

Remark 3.4: The minimization problem in (III.6) is solvable by reducing it to the Nehari problem. We can therefore check the sufficient condition in Theorem 3.3 and obtain $Q$ satisfying (III.3) by efficient matrix computations; see [10, Chapter 8] for details.

The proposition below shows that the system (II.2) always satisfies the assumptions on $D_{\theta}$ and $N_{\theta}$ in Theorem 3.3 if the plant is a single-input system with invertible $A$. We also obtain $L$ and $f$ in (III.5) without calculating a coprime factorization of $P_{\theta}$ for all $\theta \in S$.

Proposition 3.5: Suppose that $0 \in(\underline{\Delta}, \bar{\Delta})$ and that the plant (II.1) is a single-input system. Define $P_{\Delta}(z):=$ $z H_{\Delta}\left(I-z F_{\Delta}\right)^{-1} G_{\Delta}$. For all $\Delta \in(\underline{\Delta}, \bar{\Delta})$, there exists a right coprime factorization $P_{\Delta}=D_{\Delta}^{-1} N_{\Delta}$ satisfying $D_{\Delta}=D_{0}$. Furthermore, suppose that $A$ is invertible and is in Jordan canonical form. Since $B$ is a vector and since $e^{-A \Delta}$ is upper triangular, we can define

$$
\begin{aligned}
& {\left[\begin{array}{c}
\alpha_{1}(z) \\
\vdots \\
\alpha_{n}(z)
\end{array}\right]:=\left(\left(\frac{1}{z} I-\Phi\right)^{-1}(\Phi-I)+z I\right) A^{-1} B} \\
& {\left[\begin{array}{ccc}
\beta_{1,1}(\Delta) & \cdots & \beta_{1, n}(\Delta) \\
0 & \ddots & \vdots \\
0 & 0 & \beta_{n, n}(\Delta)
\end{array}\right]:=e^{-A \Delta}-I .}
\end{aligned}
$$

Then (III.5) holds with

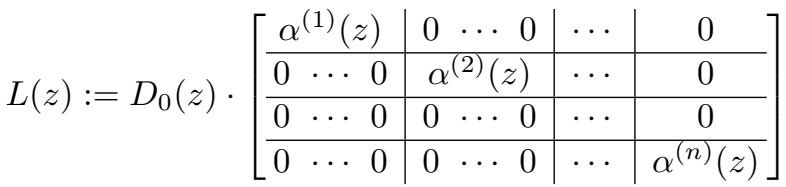

$$
\begin{aligned}
& f(\Delta):=\left[\begin{array}{llll}
\beta^{(1)}(\Delta) & \beta^{(2)}(\Delta) & \cdots & \beta^{(n)}(\Delta)
\end{array}\right],
\end{aligned}
$$

where $\alpha^{(k)}(z):=\left[\begin{array}{lll}\alpha_{k}(z) & \cdots & \alpha_{n}(z)\end{array}\right]$ and $\beta^{(k)}(\Delta):=$ $\left[\beta_{k, k}(\Delta) \cdots \beta_{k, n}(\Delta)\right]$ for $k=1, \ldots, n$.

Proof: We first obtain another realization of the system (II.2). With the similarity transformation $T$ given by

$$
T:=\left[\begin{array}{cc}
-\theta & -I \\
I+\theta & I
\end{array}\right],
$$

we can transform $F_{\Delta}$ into

$$
\bar{F}_{\Delta}:=T^{-1} F_{\Delta} T=\left[\begin{array}{ll}
p & 0 \\
0 & 0
\end{array}\right] .
$$

It follows that the eigenvalues of $F_{\Delta}$ are those of $p$ in addition to $n$ eigenvalues equal to zero, and hence the poles of the system (II.2) are independent of $\Delta$. Furthermore, if $A$ is invertible, then $G_{\Delta}$ in (II.3) is given by

$$
G_{\Delta}=\left[\begin{array}{c}
-p \theta A^{-1} B \\
(p(I+\theta)-I) A^{-1} B
\end{array}\right],
$$


and hence we have the realization $\left(\bar{F}_{\Delta}, \bar{G}_{\Delta}, \bar{H}_{\Delta}\right)$,where $\bar{G}_{\Delta}$ and $\bar{H}_{\Delta}$ are defined by

$$
\begin{aligned}
\bar{G}_{\Delta} & :=T^{-1} G_{\Delta}=\left[\begin{array}{c}
(p-I) A^{-1} B \\
\theta A^{-1} B
\end{array}\right] \\
\bar{H}_{\Delta} & :=H_{\Delta} T=\left[\begin{array}{ll}
I+\theta & I
\end{array}\right] .
\end{aligned}
$$

Since $D_{\Delta}$ is a scalar-valued function, it follows from [9, Theorem 4.32] that $z_{0} \in \overline{\mathbb{D}}$ is a zero of $D_{\Delta}$ if and only if $1 / z_{0}$ is an eigenvalue of $p$. As $D_{\Delta}$ for all $\Delta \in(\underline{\Delta}, \bar{\Delta})$, we can therefore choose an $\mathcal{R} \mathcal{H}^{\infty}$ function whose zeros are the reciprocals of the unstable eigenvalues of $p$.

Moreover, since $D_{\Delta}=D_{0}$, it follows that $N_{\Delta}-N_{0}=$ $D_{0}\left(P_{\Delta}-P_{0}\right)$. From the realization $\left(\bar{F}_{\Delta}, \bar{G}_{\Delta}, \bar{H}_{\Delta}\right)$, we see that $P_{\Delta}(z)-P_{0}(z)$ equals

$$
\left(e^{-A \Delta}-I\right)\left(\left(\frac{1}{z} I-p\right)^{-1}(p-I)+z I\right) A^{-1} B .
$$

Thus (III.7) and (III.8) give (III.5).

\section{EXaCt BOUND FOR SCAlar Systems}

The objective in this section is to reduce the problem of finding $Q \in \mathcal{R H}^{\infty}$ such that (III.3) holds for all $\theta \in S$ to a computationally verifiable one for the following unstable scalar plant: $\dot{x}=a x+b u$ with $a>0$. If the plant is stable, the stabilization problem is trivial because zero control input $u(t)=0$ leads to the stability of the closed-loop system.

The extended system (II.2) is given by

$$
\begin{aligned}
\xi_{k+1} & =p\left[\begin{array}{cc}
-\theta & -\theta \\
1+\theta & 1+\theta
\end{array}\right] \xi_{k}+\frac{b}{a}\left[\begin{array}{c}
-p \theta \\
p(1+\theta)-1
\end{array}\right] u_{k} \\
y_{k} & =\left[\begin{array}{ll}
0 & 1
\end{array}\right] \xi_{k},
\end{aligned}
$$

where $p:=e^{a h}$ and $\theta:=e^{-a \Delta}-1$. In what follows we take $b / a=1$ for simplicity of notation, because simultaneous stabilizability does not depend on this ratio.

Taking the Z-transform of (IV.1) and then mapping $z \mapsto$ $1 / z$, we obtain the transfer function $P_{\theta}$ :

$$
P_{\theta}(z)=\frac{(p-1) z}{1-p z}-\theta \frac{p z(z-1)}{1-p z} .
$$

The extended system (IV.1) is stabilizable and detectable except for $\theta=-1$, at which point the system loses detectability.

Since $\theta=e^{-a \Delta}-1$, it follows that

$$
\Delta \in[\underline{\Delta}, \bar{\Delta}] \quad \Leftrightarrow \quad \theta \in\left[e^{-a \bar{\Delta}}-1, e^{-a \underline{\Delta}}-1\right]=: S .
$$

From Assumption 2.1, the set $S$ on $\theta$ is a subset of

$$
\left(e^{-a h}-1, e^{a h}-1\right)=: S_{\max } .
$$

The system (IV.2) belongs to a class of the so-called interval systems, whose stabilization problem has been studied, e.g., in [11]-[13]. Here we shall develop a new approach based on Theorem 3.1 This approach gives an alternative proof and provides insight into the relationship between simultaneous stabilization and interval systems.

The following theorem gives the exact bound on the clock offset for scalar systems:
Theorem 4.1: Consider a set $S$ in (IV.3) of the form $S=$ $[\underline{\theta}, \bar{\theta}] \subset S_{\max }$. There exists a stabilizer of $P_{\theta}$ for all $\theta \in S$, that is, there exists $Q \in \mathcal{R} \mathcal{H}^{\infty}$ satisfying (III.3) for all $\theta \in S$ if and only if

$$
(p-1)^{2} \bar{\theta}-(p+1)^{2} \underline{\theta}<4 p .
$$

In particular, if $-\underline{\theta}=\bar{\theta}$, then (IV.5) is equivalent to

$$
\bar{\theta}<\frac{2 p}{p^{2}+1} .
$$

If we change the offset variable from $\theta=e^{-a \Delta}-1$ to $\Delta$, then (IV.3) and (IV.5) give the maximum length of the offset interval $[\underline{\Delta}, \bar{\Delta}]$ allowed by a linear time-invariant controller.

Corollary 4.2: Let $\underline{\Delta}<0<\bar{\Delta}$. There exist a stabilizer of the extended system (II.2) for all $\Delta \in[\underline{\Delta}, \bar{\Delta}]$ if and only if $\bar{\Delta}-\underline{\Delta}<2(\log (p+1)-\log (p-1)) / a$.

We prove Theorem 4.1 by reducing the simultaneous stabilization problem to the Nevanlinna-Pick interpolation problem. To this end, first we show that the stabilization problem is equivalent to an interpolation problem with a specified codomain:

Lemma 4.3: Let $\underline{\theta}<0<\bar{\theta}$ and let $S:=[\underline{\theta}, \bar{\theta}] \subset S_{\max }$. There exists a stabilizer of $P_{\theta}$ for $\theta \in S$, that is, there exists $Q \in \mathcal{R} \mathcal{H}^{\infty}$ satisfying (III.3) for all $\theta \in S$ if and only if there exists $F \in \mathcal{R H}^{\infty}$ such that $F$ is a map from $\overline{\mathbb{D}}$ to $\mathbb{C} \backslash\{(-\infty, 1 / \underline{\theta}] \cup[1 / \bar{\theta}, \infty)\}$ and satisfies the interpolation conditions $F(0)=0, F(1)=0$, and $F(1 / p)=-1$.

Proof: We obtain an $\mathcal{R} \mathcal{H}^{\infty}$ coprime factorization $P_{\theta}=$ $N_{\theta} / D_{\theta}$ with

$$
N_{\theta}(z):=\frac{(p-1) z}{z-\alpha}-\theta \frac{p z(z-1)}{z-\alpha}, \quad D_{\theta}(z):=\frac{1-p z}{z-\underset{\text { (IV.7) }}{\alpha},}
$$

where $\alpha$ is a fixed complex number with $|\alpha|>1$. If we define $X_{0}$ and $Y_{0}$ by

$$
X_{0}(z):=\frac{1-\alpha p}{p-1}, \quad Y_{0}(z):=-\alpha,
$$

then the Bezout identity $N_{0} X_{0}+D_{0} Y_{0}=1$ holds. Hence defining

$$
T_{1}(z):=p \frac{1-\alpha p}{p-1} \frac{z(z-1)}{z-\alpha}, \quad T_{2}(z):=p \frac{z(z-1)(1-p z)}{(z-\alpha)^{2}}
$$

we see that $U_{\theta}+Q V_{\theta}$ in (III.3) satisfies

$$
U_{\theta}+Q V_{\theta}=1-\theta\left(T_{1}+Q T_{2}\right) \quad\left(Q \in \mathcal{R} \mathcal{H}^{\infty}\right) .
$$

Define $S_{+}:=S \backslash\{0\}$. Theorem 3.1 and (IV.10) show that the plant $P_{\theta}$ is simultaneously stabilizable by a single stabilizer if and only if there exists $Q \in \mathcal{R} \mathcal{H}^{\infty}$ such that

$$
\left(1-\theta\left(T_{1}+Q T_{2}\right)\right)^{-1} \in \mathcal{R H}^{\infty} \quad\left(\theta \in S_{+}\right) .
$$

We have (IV.11) if and only if $1-\theta\left(T_{1}+Q T_{2}\right)$ has no zero in $\overline{\mathbb{D}}$ for all $\theta \in S_{+}$, that is,

$$
T_{1}(z)+Q(z) T_{2}(z) \in \mathbb{C} \backslash\{(-\infty, 1 / \underline{\theta}] \cup[1 / \bar{\theta}, \infty)\}
$$

for all $z \in \overline{\mathbb{D}}$.

Now we prove the equivalence between the stability of $Q$ and the interpolation conditions on $F$. 
Suppose that $Q \in \mathcal{R} \mathcal{H}^{\infty}$ and define $F$ by $F:=T_{1}+$ $Q T_{2} \in \mathcal{R} \mathcal{H}^{\infty}$. Since the unstable zeros of $T_{2}$ are 0,1 , and $1 / p$ and since $Q$ is stable, it follows that $F(0)=T_{1}(0)=0$, $F(1)=T_{1}(1)=0$, and $F(1 / p)=T_{1}(1 / p)=-1$.

Conversely, let $F \in \mathcal{R} \mathcal{H}^{\infty}$ satisfy $F(0)=0, F(1)=0$, and $F(1 / p)=-1$. If we define

$$
Q:=\frac{F-T_{1}}{T_{2}},
$$

then $Q$ belongs to $\mathcal{R} \mathcal{H}^{\infty}$. In fact, assume that $Q \notin \mathcal{R} \mathcal{H}^{\infty}$. Since

$$
Q T_{2}=F-T_{1} \in \mathcal{R} \mathcal{H}^{\infty}
$$

it follows that $Q$ has some unstable poles that are zeros of $T_{2}$ in $\overline{\mathbb{D}}$. Let $p_{0}$ be one of the poles. Since $T_{2}$ has only simple zeros in $\overline{\mathbb{D}}$, it follows that $\left(Q T_{2}\right)\left(p_{0}\right) \neq 0$. The interpolation conditions of $F$ lead to $F\left(p_{0}\right)-T_{1}\left(p_{0}\right)=0$, which contradicts (IV.13). This completes the proof.

As in [14], we solve the interpolation problem in Lemma 4.3 via the Nevanlinna-Pick interpolation. We need to a conformal map from $\mathbb{G}:=\mathbb{C} \backslash\{(-\infty, 1 / \underline{\theta}] \cup[1 / \bar{\theta}, \infty)\}$ to $\mathbb{D}$. In [12], [14], [15, Section 4.1], such a conformal map is given by

$$
\phi: \mathbb{G} \rightarrow \mathbb{D}: s \mapsto \frac{1-\sqrt{(1-\bar{\theta} s) /(1-\underline{\theta} s)}}{1+\sqrt{(1-\bar{\theta} s) /(1-\underline{\theta} s)}}
$$

The conformal map defined in (IV.14) gives the following equivalence:

Problem 4.4: Let $z_{1}, \ldots, z_{n}$ be distinct points in $\overline{\mathbb{D}}$ and let $w_{1}, \ldots, w_{n}$ belong to $\mathbb{G}$. Find a function $F$ such that

- $F: \overline{\mathbb{D}} \rightarrow \mathbb{G}$,

- $F$ is holomorphic in $\mathbb{D}$ and continuous in $\overline{\mathbb{D}}$, and

- $F\left(z_{i}\right)=w_{i} \quad(i=1, \ldots, n)$.

Lemma 4.5: Problem 4.4 is equivalent to the problem of finding a function $G$ such that

- $G: \overline{\mathbb{D}} \rightarrow \mathbb{D}$,

- $G$ is holomorphic in $\mathbb{D}$ and continuous in $\overline{\mathbb{D}}$, and

- $G\left(z_{i}\right)=\phi\left(w_{i}\right) \quad(i=1, \ldots, n)$.

Proof: This follows from the fact that $G=\phi \circ F$. Finally we obtain the proof of Theorem 4.1.

Proof of Theorem 4.1: Lemmas 4.3 and 4.5 show that the stabilization problem with clock offsets can be reduced to the Nevanlinna-Pick interpolation problem with a boundary condition [15, Chapter 2]. We therefore obtain a necessary and sufficient condition based on the positive definiteness of the associated Pick matrix, which is equivalent to (IV.5). The detailed calculation is omitted due to space constraints.

Remark 4.6: Since

$$
\phi^{-1}(s)=\frac{4 s}{\bar{\theta}(s+1)^{2}-\underline{\theta}(s-1)^{2}}
$$

and since $F=\phi^{-1} \circ G$, it follows that $F$ is rational for every rational solustion $G$ of the Nevanlinna-Pick interpolation problem. From (IV.12), we can therefore construct a rational function $Q=\left(\phi^{-1} \circ G-T_{1}\right) / T_{2}$ satisfying (III.3) and a finite-dimensional stabilizer of the extended system (II.2) for every $\Delta \in[\underline{\Delta}, \bar{\Delta}]$.

\section{Comparison in the Case of Scalar Plants}

In this section, the bounds on the clock offset that can be allowed using a linear time-invariant stabilizer (obtained using Theorems 4.1 and 3.3) are compared with the exact bound that would be allowed by a static state feedback stabilizer, and with a sufficient condition on offset ranges for the existence of 2-periodic static stabilizers that makes (IV.1) stable. We also obtain a bound obtained by robust control that regards the clock offset as an additive uncertainty.

A. Comparison with static stabilizers and 2-periodic static stabilizers

The proposition below gives the exact bound on the clock offset that could be obtained using a static stabilizer for a scalar plant. A sufficient condition on offset ranges is also provided for the existence of 2-periodic stabilizers that stabilize the extended system (IV.1).

Proposition 5.1: Consider the extended system (IV.1). Define $p:=e^{a h}, \theta:=e^{-A \Delta}-1$, and $S_{\max }$ as in (IV.4). There exists a static stabilizer $u_{k}=-K y_{k}$ that makes the extended system (IV.1) stable for every $\theta \in S \subset S_{\max }$ if and only if $S$ satisfies

$$
S \subset\left(-\frac{1}{p}, \frac{1}{p}\right) .
$$

Furthermore, There exists a 2-periodic static stabilizer

$$
\left[\begin{array}{c}
u_{k} \\
u_{k+1}
\end{array}\right]=-\left[\begin{array}{cc}
K_{1} & 0 \\
0 & K_{2}
\end{array}\right]\left[\begin{array}{c}
y_{k} \\
y_{k+1}
\end{array}\right]
$$

that makes the extended system (IV.1) stable for every $\Delta \in$ $S \subset S_{\max }$ if

$$
S \subset\left(-\frac{1}{p \kappa}, \frac{1}{p \kappa}\right)
$$

where

$$
\kappa:=\frac{\sqrt{p^{2}+1} \sqrt{p^{2}-4 \sqrt{2}+5}-2(\sqrt{2}-1) p}{p^{2}-1}<1 .
$$

Proof: The proof is based on the Jury stability criterion, but is omitted due to space constraints.

Comparing (IV.6), (V.1), and (V.3), we see from a routine calculation that for all $p>1$,

$$
\frac{1}{p}<\frac{1}{p \kappa}<\frac{2 p}{p^{2}+1} .
$$

As expected, (V.1) results in the smallest range for values of $\theta$ because the set of all static stabilizers is a subset of the class of linear time-invariant stabilizers and that of 2-periodic static stabilizers in (V.2). On the other hand, 2-periodic static stabilizers may not belong to the class of linear time-invariant stabilizers, and vice versa. The second inequality in (V.4) always holds for all $p>1$, but the bound (V.3) is a sufficient condition. In order to compare the performance of 2-periodic static stabilizers and time-invarinat stabilizers, we need to do a brute-force computation for the exact bound on the clock offset that would be allowed by a 2-periodic static stabilizer. 


\section{B. Sufficient condition from Theorem 3.3}

From (IV.7), we see that $D_{\theta}=D_{0}$ and $N_{\theta}(z)-N_{0}(z)=$ $(\theta p z(z-1)) /(z-\alpha)$. Theorem 3.3 shows that there exists a stabilizer of $P_{\theta}$ for all $\theta$ with $|\theta|<1 / p$. The bound is the same as (V.1) by a static stabilizer. This confirms that the use of the small gain theorem instead of the conformal map $\phi$ leads to a conservative stabilization analysis.

\section{Sufficient condition from robust control of plants with additive uncertainties}

Take $P_{0}=(p-1) z /(1-p z)$ as a nominal plant. An arbitrary plant $P_{\theta}$ could be viewed as a perturbation of $P_{0}$ by the following additive uncertainty: $P_{\theta}(z)-P_{0}(z)=$ $m(z) W_{\theta}(z)$, where $m(z):=z(p-z) /(1-p z)$ and $W_{\theta}(z):=$ $\theta p(z-1) /(p-z)$. Since $\left|m\left(e^{j \omega}\right)\right|=1$, it follows that $\left|P_{\theta}\left(e^{j \omega}\right)-P_{0}\left(e^{j \omega}\right)\right| \leq\left|W_{\bar{\theta}}\left(e^{j \omega}\right)\right|$ for all $e^{j \omega} \in \mathbb{T}$ and $\theta \in[-\bar{\theta}, \bar{\theta}]$ with $\bar{\theta}>0$. Hence there exists a stabilizer of $P_{\theta}$ for all $\theta \in[-\bar{\theta}, \bar{\theta}]$, if $\bar{\theta}$ satisfies

$$
\left\|W_{\bar{\theta}}\left(X_{0}+Q D_{0}\right)\right\|_{\infty}<1
$$

for some $Q \in \mathcal{R} \mathcal{H}^{\infty}$, where $X_{0}$ and $D_{0}$ are defined as in (IV.8) and (IV.7). Reducing the problem of finding $Q \in \mathcal{R H}^{\infty}$ with (V.5) to the Nevanlina-Pick interpolation problem as in [16], we see that $\bar{\theta}$ satisfies (V.5) for some $Q \in \mathcal{R H}^{\infty}$ if and only if $\bar{\theta}<1 / p$. The bound derived from the conventional approach of robust control is also the same as (V.1) by a static stabilizer. Since in conventional robust control, we consider unnecessarily large class of uncertainties, it is not surprising to discover that this approach becomes conservative.

\section{Numerical example}

Consider a sclar plant $\dot{x}=a x+u$ and set the unstable pole $a=1$ and the sampling period $h=1$. The bound obtained using a linear time-invariant stabilizer is $-0.6321<$ $\theta<0.7227$ and hence $-0.5439<\Delta<1$. The necessary condition in Proposition 3.2 does not give any bound on $\theta$ in this example.

Fig. 3 shows the bounds on $\theta$ that can be allowed using each class of stabilizers. It may be possible that periodic dynamic stabilizers achieve better performance as in [17]. However, a numerical computation shows that there does not exist a 2-periodic static stabilizer

$$
\left[\begin{array}{c}
u_{k} \\
u_{k+1}
\end{array}\right]=-\left[\begin{array}{cc}
K_{1} & 0 \\
K_{3} & K_{2}
\end{array}\right]\left[\begin{array}{c}
y_{k} \\
y_{k+1}
\end{array}\right]
$$

that stabilizes $P_{\Delta}$ for $-0.5018<\Delta<1$ (note that $K_{3}$ in (V.6) may be nonzero). It would be an interesting topic left for future works is the problem of finding the offset bound allowed by using periodic dynamic stabilizers.

\section{CONCLUDING REMARKS}

We studied the problem of stabilizing networked control systems with time-invariant clock offsets. We formulated the problem as the stabilization problem for systems with parameter uncertainty and derived computationally attractive conditions that are either necessary or sufficient, based on

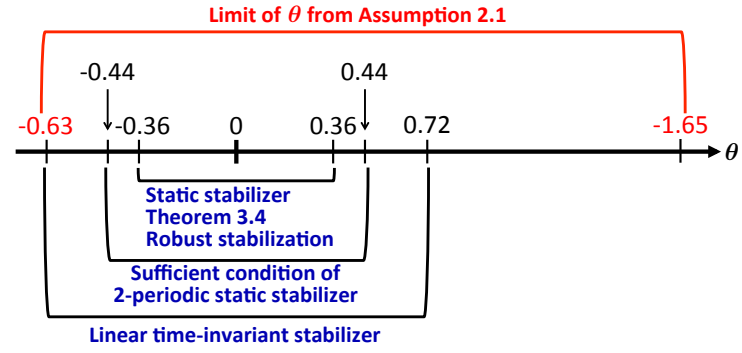

Fig. 3: Comparison among the bounds on $\theta$ obtained using each class of stabilizers

the results of simultaneous stabilization. For scalar systems, using the Nevanlinna-Pick interpolation, we obtained the exact bound on the clock offset that would be allowed by a linear time-invariant controller. However, a full investigation of the problem for general-order systems is still an open area for future research.

\section{REFERENCES}

[1] S. Graham and P. R. Kumar, "Time in general-purpose control systems: The Control Time Protocol and an experimental evaluation," in Proc. 43rd IEEE CDC, 2004.

[2] B. Sundararaman, U. Buy, and A. D. Kshemkalyani, "Clock synchronization for wireless sensor networks: a survey," Ad Hoc Networks, vol. 3, pp. 281-323, 2005.

[3] I.-K. Rhee, J. Lee, J. Kim, E. Serpedin, and Y.-C. Wu, "Clock synchronization in wireless sensor networks: An overview," Sensors, vol. 9, pp. 56-85, 2009.

[4] N. M. Freris, S. R. Graham, and P. R. Kumar, "Fundamental limits on synchronizing clocks over network," IEEE Trans. Automat. Control, vol. 56, pp. 1352-1364, 2011.

[5] X. Jiang, J. Zhang, J. J. Harding, B. J. Makela, and A. D. DomínguesGarcía, "Spoofing GPS receiver clock offset of phasor measuremen units," IEEE Trans. Power Systems, vol. 28, pp. 3253-3262, 2013.

[6] C. Bonebrake and L. R. O'Neil, "Attacks on GPS time reliability," IEEE Secur. Priv., vol. 12, pp. 82-84, 2014.

[7] K. Okano, M. Wakaiki, and J. P. Hespanha, "Real-time control under clock offsets between sensors and controllers," To appear in Proc. HSCC 2015.

[8] M. Vidyasagar and N. Viswanadham, "Algebraic design techniques for reliable stabilization," IEEE Trans. Automat. Control, vol. 27, pp 1085-1095, 1982

[9] M. Vidyasagar, Control System Synthesis: A Factorization Approach. MIT Press, Cambridge, 1985,

[10] B. A. Francis, A Course in $H_{\infty}$ Control Theory. Springer, New York, 1987.

[11] B. K. Ghosh, "An approach to simultaneous system design. Part II: Nonswitching gain and dynamic feedback compensation by algebraic geometric methods," SIAM J. Control Optim., vol. 26, pp. $919-963$ 1988

[12] A. W. Olbrot and M. Nikodem, "Robust stabilization: Some extensions of the gain margin maximization problem," IEEE Trans. Automat. Control, vol. 39, pp. 652 - 657, 1994.

[13] C. T. Abdallah, P. Dorate, F. Perez, and D. Docampo, "Controller synthesis for a class of interval plants," Automatica, vol. 31, pp. 341 - 343, 1995.

[14] P. P. Khargonekar and A. Tannenbaum, "Non-Euclidian metric and the robust stabilization of systems with parameter uncertainty," IEEE Trans. Automat. Control, vol. 30, pp. 1005-1013, 1985.

[15] C. Foiaş, H. Özbay, and A. Tannenbaum, Robust Control of Infinite Dimensional Systems: Frequency Domain Methods. Springer, London, 1996.

[16] H. Kimura, "Robust stabilizability for a class of transfer functions," IEEE Trans. Automat. Control, vol. 29, pp. 788-793, 1984.

[17] P. P. Khargonekar, K. Poolla, and A. Tannenbaum, "Robust control of linear time-invariant plants using periodic compensation," IEEE Trans. Automat. Control, vol. 30, pp. 1088-1096, 1985. 